Rojas, C. (2016). De la autogestión comunitaria a lo sentipensante. Revista de Sociología y Antropología: VIRAJES, 18 (2), 65-74. DOI: 10.17151/rasv.2016.18.2.5

\title{
DE LA AUTOGESTIÓN COMUNITARIA A LO SENTIPENSANTE
}

\section{CARLOS ARTURO ROJAS PÉREZ*}

Recibido: 30 de Marzo de 2016

Aprobado: 29 de Mayo de 2016

Artículo de Reflexión

\footnotetext{
* Historiador de la Universidad Nacional de Colombia, Diseñador Gráfico de la Corporación Unificada de Educación Superior, Magister en Museología y Gestión del Patrimonio de la Universidad Nacional de Colombia. Miembro del grupo de Investigación de Museología Aplicada del Instituto de Investigaciones Estéticas de la Facultad de Artes de la Universidad Nacional de Colombia. Museógrafo Museo de Bogotá, Instituto Distrital de Patrimonio Cultural. Diseñador CURA Soluciones Museográficas LimaPerú. caarojaspe@unal.edu.co. (ㄱ) ORCID: 0000-0002-8454-4296
} 


\title{
Resumen
}

El presente articulo busca poner en discusión el concepto del sentipensante, del sociólogo colombiano Orlando Fals Borda, con algunas de las herramientas de la Psicología Social, principalmente la que tiene que ver con la autogestión. Dicho dialogo se da metodológicamente en un contexto geográfico y temporal especifico, en una experiencia comunitaria que se desarrolla en el sur de Bogotá. Dicha experiencia permite dilucidar como puede ser la aplicación real del concepto del sentipensante como contraposición a un sistema económico y político que pretende la desestructuración de las formas de organziación autonomas y comunitarias.

Palabras clave: sentipensante, tiguaque, autogestión, glocalización.

\section{FROM THE SELF-MANAGEMENT TO THE “SENTIPENSANTE”}

\begin{abstract}
The present paper seeks to put in dialogue the concept of the "sentipensante", colombian sociologist Orlando Fals Borda, with some tools of the Social Psychology, mainly what has to do with self - management. This dialogue is given methodologically in a specific geographical and temporal context, in a community experience that is developed in the south of Bogota. This experience allows us to elucidate the real application of the contraposition to an economic and political system that aims to the destructuring of autonomous and communitarian forms of organization.
\end{abstract}

Key words: sentipensante, tiguaque, self-management, glocalization. 
1 Profesor Fals Borda fue uno de los intelectuales más
importantes para las Ciencias Humanas del siglo XX, e incluso aulas de clase, sino desde las comunidades humanas, le dieron herramientas suficientes para formular diversas soluciones con las que el país podría encontrar el rumbo, en este pequeño escrito intentaremos profundizar en algunas de sus propuestas, intentando aterrizarlas a un proceso vivido por el autor de este escrito, tal vez en un intento por contagiarse del positivismo del profesor Borda en cuanto a la posibilidad de reconstruir una nación, cada vez más, en crisis.

\section{De la huerta a la autogestión y contra la globalización}

Hace algunos días, por invitación de un compañero del Departamento de Historia, llegué a un barrio en la periferia de Bogotá, de esos barrios que no se logran ver desde la ciudad y que sin embargo desde ellos se puede contemplar toda la mole de cemento que es la capital del país. El Barrio tiene nombre con sabor indígena, no suena a santo católico o a prócer criollo, se llama Tiguaque, un poco más allá de Juan Rey, en el extremo sur oriental de la ciudad, clavado en lo majestuoso de los cerros orientales y a unos pocos kilómetros del pie de monte llanero. En ese lugar nos encontramos con una señora, de unos cuarenta años, que tenía en su casa un jardín infantil, allí nos recibió junto con su pequeña niña de ojos azules. En aquel jardín, en medio de una cuadra inclinada sin el más mínimo asomo de pavimento y a unas pocas cuadras del final de la ciudad, se reunieron varios jóvenes estudiantes, líderes comunales, y personas del barrio, en una Minga, organizada por varios colectivos dentro de los que se cuenta La Olla Artística. Todos eran conocidos, y no había distinción entre gente del barrio, de universidades públicas y otros líderes comunales, los desconocidos éramos los que por primer vez asistíamos a una Minga de cualquier tipo, y que a decir verdad estamos más acostumbrados a las bibliotecas que a las comunidades activas. El objetivo de la jornada era la construcción de un invernadero, en medio de un pequeño lote abandonado; la adaptación de la tierra de dicho lote para plantar cultivos comunitarios de legumbres y hortalizas; y la cosecha de lo que ya se había sembrado hace algunos meses y con ello hacer el almuerzo de ese día en una Olla Comunitaria. Más allá de cualquier pretensión el objetivo de quienes se han organizado en dicha comunidad no es otro que el de crear un pequeño sitio en el que la huerta, como factor practico, sirva para la construcción de comunidad y tal vez, el objetivo más ambicioso, el inicio de una especie de soberanía alimentaria que tenga su principal raíz en la vuelta al campo desde lo que la ciudad marginaliza. ¿A qué voy con 
toda esta anécdota? a Fals Borda y el nuevo compromiso de los académicos en nuestro contexto.

En el año 2003, el Ancora Editores en compañía con Panamericana, editaron una serie de ponencias del profesor Fals Borda, que habían sido recopiladas en las ultimadas décadas del autor, y que en buena parte resumían los planteamientos del Profesor en cuanto al que hacer frente a la, cada vez más, innegable crisis del país, el libro se llamó Ante la Crisis del País, Ideas-Acción para el cambio (Fals Borda, 2003). Todo el texto, si se mira en conjunto, tiene como objetivo sentar las bases para lo que sería una Segunda República, ante el fracaso de la Primer República que se inició en el siglo XIX. Esta Segunda República tendría como fundamento la endogénesis, concepto que el profesor Borda utiliza para designar una especie de movimiento que tendría que hacer la nación sobre sí misma, para empezar a reconstruirse desde lo autóctono en conjunto, es decir, desde lo que en verdad es Colombia, tanto lo que está en el territorio desde hace 12.000 y tantos años, hasta lo que nos llegó de oriente, y nos sigue llegando, hace 500 años, dándole primacía a lo nativo, que es esa parte de Colombia, que pese al mestizaje, aún pervive con ese arraigo a la tierra más que al capital. La propuesta del profesor Borda es un llamado al equilibrio entre lo moderno y lo ancestral. Esto se propone a través de cinco premisas; la creación de una unidad nacional desde lo diverso (la cual en parte logro tener cabida en la Constitución de 1991); la construcción desde lo regional (Borda insiste en la obsolescencia de los departamentos como unidades administrativas, dándole primacía a las regiones orgánicas); darle relevancia al pensamiento ancestral indígena; sumar dicho saber al saber académico; y por último la creación de un ecosocialismo fundamentado en la resolución de problemáticas propias y no como imposición de formular extranjeras. Estas premisas logran condensar ese sistema de equilibrio que podría hacer funcionar de nuevo unas dinámicas sociales saludables y más humanas.

Es interesante ver que el profesor Borda pide, en el último escrito del libro, aquel en el que recibe la Orden Gerardo Molina de la Universidad Nacional, que estas teorías planteadas por la academia no solo sean teorías, sino que los académicos sean los principales propulsores de llevarlas a la praxis, transformando estos planteamientos en verdaderas ideas-acción, para esto es necesario compromiso y activismo político, no dogmatismo, sino activismo.

Pues bien, allí estábamos con las manos cansadas de usar, en medio de nuestra ignorancia, un azadón, unas palas, machetes y manos, para limpiar la tierra, hacer surcos e intentar cultivar algo productivo, así nos dio el final de la tarde, tiempo después empecé a ver en ese día la aplicación 
de las tesis del profesor Borda; no eran académicos teorizando, eran académicos produciendo, creando una comunidad que no tenía su piedra angular en ningún sistema oficial o comercial, sino propio. Allí se volvía al campo, en medio de la ciudad, lo saberes adquiridos por las señoras de mayor edad, habitantes del barrio, se ponían en práctica, pues eran ellas quienes decían como se sembraba, los ignorantes citadinos obedecía(mos) mientras aprendía(mos), sin libros, sin teoría, sin andamiajes academicistas o científicos. ¿Qué logran este tipo de actos como esta Minga a largo plazo? ¿De qué naturaleza son estos pequeños movimientos?

Empecemos por responder la última pregunta; el proceso llevado a cabo en el Barrio Tiguaque por este colectivo de la Olla Artística, entre otros grupos, es un digno proceso de Autogestión Comunitaria, sobre el que ampliamente ha teorizado la Psicología Comunitaria. Según esta rama de la psicología la Autogestión se puede definir como:

Todas las opciones de auto organización social y comunitaria donde la comunidad misma, ya sea sindical, cooperativa, campesina, de mujeres, jubilados, marginados y de cualquier otro sector social oprimido en nuestra sociedad, tome en sus propias manos la tarea de resolver sus necesidades. Para ello se tienen una serie de principios prácticos que encierran el funcionamiento básico de una sociedad autogestionaria: 1.democracia directa, 2. acción directa, 3. apoyo mutuo, 4. extensión y 5. Formación. (Brivio, 2001)

En un enfoque aún más global, la Autogestión Comunitaria, es una de las principales herramientas de la Psicología Comunitaria, la cual nació en un ambiente de caída de paradigmas, en los 60. Uno de estos paradigmas sostenía a la psicología como método para sanar lo que no está sano, pero este nuevo enfoque esta direccionado a potencializar las capacidades de lo humano, desde sí mismo, para lograr su realización. Estas capacidades no solo se refieren a lo individual, sino a lo social. Según esto, los procesos de Autogestión, como los describe esta nueva rama de la psicología tienen las siguientes características:

En primer lugar se gobierna, $u$ ordena, a modo de asamblea horizontal, donde debe haber una mezcla necesaria entre el saber popular y el saber técnico, o académico (Montero, 2008). Aquí vemos una de las tesis propuestas por Borda de amalgama entre lo ancestral y lo académico.

En segundo lugar debe haber un liderazgo democrático, lo cual genera menos dependencias en lideres (Rodrigues, 1983), bien sean autoproclamados o seguidos por su carisma o poder tradicional, como lo plantearía Weber. 
Una tercera medida es la de contemplar los procesos de Autogestión como proyectos a largo plazo, entendiendo que en la "concretitud" del fin se logra lo concreto del proyecto (Brivio, 2001).

Por último está la necesidad de que todos los miembros de la comunidad participen, es decir, que la autogestión tiene una naturaleza de inclusión, y no de exclusión. (Rodrigues, 1983).

Este tipo de estrategias lograrían la creación de una sociedad en red, similar a la que propone el profesor Borda. Sociedades que se fundamentan en la diversidad y la pluralidad de los miembros que la componen (como en el caso de Tiguaque: locales, estudiantes, líderes comunales, niños, invitados, madres cabeza de familia, etc.), Esta diversidad ofrece multidimensionalidad en la intervención y en los métodos (Montero, 2003), creando un ambiente de participación, compromiso, construcción colectiva, interdependencia, democratización, afectividad y solidaridad (Montero, 2003), además de generar procesos de apropiación y auto identificación comunitaria "Esto supone un hacerse fuerte para transformar un entorno, transformándose a sí mismos" (Montero 2003).

A si mismo se prevé un proceso para lograr dicha Autogestión, y los beneficios que por medio de esta se consolidan: En primera medida el desarrollo del sentido "ser-en-relación-con-el-mundo"; Sumado a la compresión cada vez más crítica de los problemas sociales y comunales; para así llegar al diseño de estrategias y recursos funcionales para la consecución de roles sociopolíticos personales y colectivos (Kieffer, 1982). Es decir; que desde la concepción clara del rol del yo en el mundo, en el ecosistema o en el ambiente, se llega a una concepción reflexiva del rol de ese yo con el mundo, para desde esa relación construir estrategias sociales y políticas. Todo este proceso se puede resumir con el bonito termino que el profesor Borda rescata de la cultura momposina; el sentipensante, aquel ser que vive según el equilibrio que le impone el sentimiento y el pensamiento. Si a esto se le suma que ese yo que es guiado por la razón y la emocionalidad no puede ser si no es construido por su otro, tenemos que no solo es un ser sentipensante en cuanto a si mismo, sino que debe serlo también en relación a su comunidad, desde donde empieza el trabajo de la sociedad en red, de la nación en red.

Partiendo de lo anterior podríamos responder a la primer pregunta ¿Qué lograría un proceso como el de esta Minga en el barrio Tiguaque?, en principio, no mucho, sin embargo si se entiende que los procesos de Autogestión son de largo plazo, podríamos ver la potencialidad que tiene este evento en lo que Borda llamaría la construcción de la Segunda República. La soberanía alimentaria, como principio y objetivo de este tipo de procesos de agricultura urbana, lograría poner en la mesa una de las cartas más 
importantes del país frente a los procesos de globalización, procesos que a fin de cuentas pretenden la homogenización de los pueblos por reglas de mercado, valga la pena decirlo, capitalista, la carta de la agricultura auto sostenible. Este mercado, el global, tiene como principio la monetización de la economía y el enlazamiento de las economías locales a una economía global, incluyendo la explotación de las riquezas comunales, en este caso agrícolas, para el beneficio de grupos macro, bien sean naciones, regiones transnacionales o incluso el globo como tal. Según esto la famosa Aldea Global termina por ser una espiral sin fondo en la que los recursos naturales locales se pierden en un mercado cada vez más gigantesco, además de entender que dicho mercado es el que pone las reglas; los precios, los medios y métodos de distribución, y hasta el tipo de consumidores, que tendrían dichos productos agrícolas. Es decir, para resumir, el mercado global de alimentos seria el que impondría, en las dinámicas de la globalización, que, como, cuando y donde se cultivan los alimentos. Pero si en este escenario las comunidades urbanas, y rurales, lograran crear procesos de autogestión comunitaria, entendiese a sí mismas como una amalgama de individuos plurales, se podría imponer una dinámica diferente desde lo local, hacia lo regional y lo nacional, y de ahí a lo global, es esto a lo que hace referencia el profesor Borda cuando esgrime el concepto de Glocalización, lo cual traduce que desde lo local se imponga el ritmo a lo global, y no al contrario. Se podría decir, en este orden de ideas, que la Autogestión Comunitaria, del estilo Minga en Tiguaque, puede ser la piedra angular para hacer frente a la cada vez más imponente y homogenizante Globalización.

\section{De la autogestión, los Estados y los sentipensantes}

En 1990, Charles Tilly, desde la sociología histórica, hacia un importante aporte en cuanto a la definición de los Estados, si bien se refiera a Europa, sus tesis sobre la construcción de un Estado se podían extrapolar a cualquier parte del mundo, en cuanto a que todos los Estados tienen como modelo o Norteamérica o Europa. Tilly mostraba como los Estados, que se definen, a la manera de Weber, como aquellas instituciones de dominación que logran monopolizar el control de la fuerza, en una serie de etapas, desde el patrimonialismo hasta la especialización de las burocracias, pero por dos vías separadas; en primer lugar estaba la vía de la coerción, la cual se fundamentaba en la acumulación y concentración de medios coercitivos, con predominio en regiones rurales; en segunda medida estaba la vía del capital, que no era otra cosa que la acumulación y concentración del capital, con prelación en zonas densamente urbanizadas; por ultimo estaba una extraña simbiosis entre estas dos vías que se resumía en la vía de la coerción 
capitalizada, que se definiría como una especie de camino intermedio entre la acumulación de capital y de coerción. (Tilly, 1990), La tercera vía, la más apropiada para la formación de Estados modernos, fue la que los Estados más exitosos tomaron, dando así una adjetivo a los Estados de Estados mafiosos, por ser instituciones, que en pasando por encima de cualquier cosa, se auto sostenían en mantener guerras externas, ya que dichas guerras no solamente estructuraban parte de la burocracia estatal, sino que creaban el Estado como tal, por ser la guerra la principal excusa y objetivo de la captación de recursos y la posterior acumulación de estos.

Si el modelo se traslada a Colombia tenemos este no se adaptaría de forma perfecta al modelo, empezando por que Colombia poco o nada a guerreado con otros países que no sean España, o mediocremente con Perú, además de saber de antemano que Colombia es todo menos un Estado exitoso. Sin embargo algunos elementos si se logran acoplar al modelo colombiano, en cuanto a que, tal vez sin pensarlo, es un país que logra andar por una vía de coerción capitalizada de manera distinta.

La constante en Colombia desde el Siglo XIX es el conflicto interno, agravado en los años 50 del siglo XX y consolidado, además de reconocido, en las últimas décadas, para nadie es un secreto que dicho conflicto ha generado un discurso maniqueista que plantea una constante lucha entre el bien (el gobierno) y el mal (grupos subversivos), discurso que ha dado para la activación e implementación de una maquinaria guerrerista que deja a Colombia con un ejército de similares proporciones al ejército brasilero, entendiendo la inmensidad del país vecino. Este discurso a dado, en los últimos cuatro gobiernos, el sustento necesario para que el Estado, y sus gobernantes, subsistan y se mantengan. Es decir que según esto, podríamos plantear que la vía de la coerción de Tilly la tenemos, pero con una guerra interna y que paradójicamente dicha vía se ha dado por que el monopolio de las armas no es enteramente del Estado, pero según esto es cada vez más obvio que mantener la Guerra significa mantener el Estado, por lo cual cualquier iniciativa de paz por parte del gobierno es más bien discursos, electorales, populistas y mediáticos que en poco o nada se reflejarán en la realidad.

En cuanto a la vía capitalista, también la tenemos, ya que es sabido que Colombia es uno de los países con más altos índices Gini, es decir que acumulación hay, y que en los últimos gobiernos, que han tenido por lema la "confianza inversionista" que se traduce en la fuerte acumulación de capitales por parte de empresas extranjeras, y algunos oligopolios nacionales, que van en crecimiento. Sin embargo, a diferencia de lo planteado por Tilly, no es el Estado propiamente dicho quien maneja dicha acumulación. Lo cual ha dejado al mismo Estado en una posición débil, casi 
inoperante de no ser para la guerra. En esta truncada dinámica se mueve el país, al que se le suman los procesos de globalización, que con ejemplos tan claros como el TLC, muestran el interés de pensar Colombia desde el mercado internacional. ¿Cómo quedan jugadas las tesis de Fals Borda para la construcción de la Segunda República en este panorama?

Es claro que los procesos de Autogestión que se proponen como forma metodológica para las tesis teóricas de Fals Borda no la tienen nada fácil en un país construido para la Guerra, las mismas propuestas de Fals Borda parecen no ser tenidas en cuenta. Justo unos pocos años después de la muerte del profesor, el país se enfrentaba al boom del TLC, en el cual la economía colombiana, y por ahí derecho la cultura y lo social, dejan de pensarse desde lo local para pensarse desde lo global. Y si miramos la insistencia de unas clases políticas que ven en las guerrillas solo un enemigo al cual destruir, imponiendo un discurso que divide al país en dos bandos, asistimos a la imposición de los modelos de Estado de Estados Unidos y Europa basados en la guerra como construcción de Estado, modelo que valga la pena decirlo las mismas guerrillas sostienen aparentemente sin saberlo, imponiéndose sin tregua a una sociedad a la que cada vez más se le aplastan sus valores ancestrales, no solo los valores indígenas sino incluso los valores cristianos, que el mismo Borda propone como herramienta útil para la construcción del país desde la endogénesis. En resumen el modelo de la glocalización, no parece tener cabida en un país hecho para la guerra, presupuestado (casi literalmente) para un conflicto que no se puede acabar con el accionar bélico, un país que no se piensa a sí mismo, sino que lo piensan desde afuera, donde se localiza la acumulación de capital a costa de los recursos naturales que le son progresivamente arrebatados a las comunidades que no tienen más elección que empezar a autogestionarse como seres sentipensantes políticamente activos, no desde las armas, sino desde la tierra, no desde los dogmas, sino desde el sentimiento profundo que afianza comunidades y regiones. Solo en esta medida se podría pensar incluso en la resolución del conflicto, ya que cualquier propuesta que venga desde lo oficial equivaldrá a sostenerlo ¿Por qué?, por que como vimos con Tilly, los Estados, al modo Europeo, se sostienen y se crean con la guerra, no con la paz.

\section{A modo de conclusión}

Se ha pretendido, grosso modo, de profundizar en el principal concepto del Profesor Borda en el texto ya mencionado, concepto que apunta a la construcción desde lo propio, desde esa reflexión hemos intentado apuntalar la tesis de la autogestión comunitaria como herramienta para la 
construcción de comunidades que se autopiensen según su diversidad y según sus entornos y problemas, haciendo una profunda retroalimentación entre academia y saber popular, para lo cual se planteó el ejemplo de la Minga comunitaria del barrio Tiguaque, ejemplo de autogestión a pequeña escala pero con una premisa ambiciosa de soberanía, que a la postre, si dicho módulo se repitiera y se logrará consolidar en supermódulos de autogestión, estaríamos a portas de una verdadera glocalización.

Dicho proceso serviría para hacer frente a un Estado que se construye desde la guerra como argumento discursivo y organizativo, construcción con bases en modelos extranjeros (De derecha o de izquierda) y que solo agravarían aún más la crisis de una nación que debe refundarse desde el trópico, desde lo andino, desde lo campesino, desde lo sentipensante.

\section{Referencias bibliográficas}

Brivio A. La Autogestión comunitaria (2001, febrero). Gestiópolis [en línea]. Bogotá, Colombia: Consultado el: 12 de abril de 2011, dehttp://www.gestiopolis.com/recursos/ documentos/fulldocs/eco/autogescomuni.htm

Fals Borda, Orlando. (2003). Ante la crisis del país, ideas-acción para el cambio. El Ancora Editores, Panamericana Editorial. Bogotá.

Kieffer, J. (1982): “The development of empowerment: the development of participatory competence among individuals in citizen organizations", Division 27 News- letter, 76(1). Estados Unidos.

Montero, M. (2003) Construcción y Crítica de la Psicología Social. Buenos Aires: Editorial Paidós.

Montero, M. (2008). Teoría y Práctica de la Psicología Comunitaria. La Tensión entre Comunidad y Sociedad. Buenos Aires: Editorial Paidós.

Musitu, O., Herrero, J. Cantera, L. y Montenegro, M. (2004). Introducción a la Psicología comunitaria. Barcelona: Editorial OUC.

Rodrigues, A. (1983). Aplicaciones de la Psicología Social. México: Editorial Trillas.

Tilly, Charles (1990). Coerción, Capital y Estados Europeos, 990-1990. Alianza Editores. Madrid 